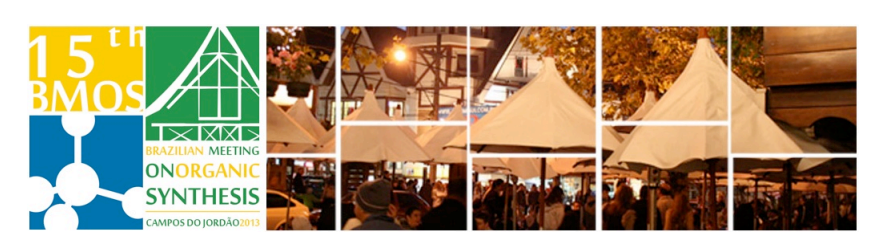

\title{
Formal $\gamma$-alkynylation of ketones via Pd-catalyzed C-C cleavage
}

\section{Asraa Ziadi, Arkaitz Correa and Rubén Martin*}

Av. Països Catalans 16, 43007 Tarragona, Spain

rmartinromo@iciq.es; aziadi@iciq.es

\section{$\beta$-Carbon Elimination; $\gamma$-Alkynylation; Palladium-Catalyzed}

\section{INTRODUCTION}

An efficient $\mathrm{Pd}$-catalyzed ketone $\gamma$-arylation via $\beta$ carbon elimination ${ }^{1}$ with aryl chlorides, was recently developed in our group (Figure 1). ${ }^{2}$ Encouraged by these results, and due to the prevalence of versatile alkyne motifs in numerous bioactive compounds and electronic materials, we set out to investigate the $\gamma$ alkynylation of cyclobutanols (Figure 1 ). ${ }^{3}$

Figure 1. Synthesis of $\gamma$-Functionalized ketones

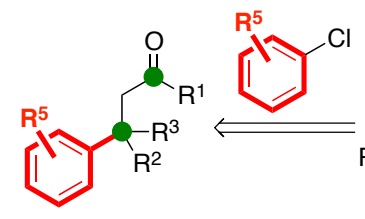

up to $99 \%$ isolated yield (28 examples)
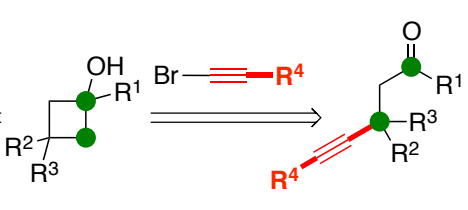

$35-92 \%$ isolated yield (20 examples)

\section{RESULTS AND DISCUSSION}

After considerable optimization, we found that the combination of $\mathrm{Pd}(\mathrm{OAc})_{2}$, SPhos and $\mathrm{Cs}_{2} \mathrm{CO}_{3}$ in toluene provided the best results for the coupling reaction of tert-cyclobutanols with (bromoethynyl)triisopropylsilane (Figure 2). As presented, the reaction manifests a broad substrate scope in which both aromatic and aliphatic groups at different positions on the tert-cyclobutanol backbone.

Figure 2. Scope of the reaction
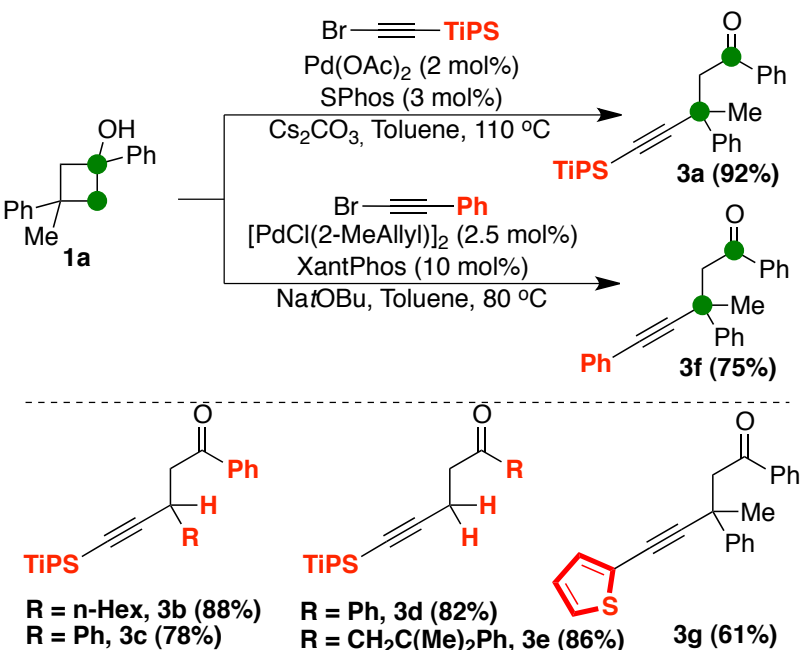

We found that, the combination of $[\mathrm{PdCl}(2-\mathrm{MeAllyl})]_{2}$, Xantphos and $\mathrm{NatOBu}$ in toluene provided the best results when using non-silylated bromoacetylene derivatives.

As shown in figure 3 , we demonstrate that this protocol could serve as a platform for molecular diversity (Figure 3).

Figure 3. Synthetic applicability
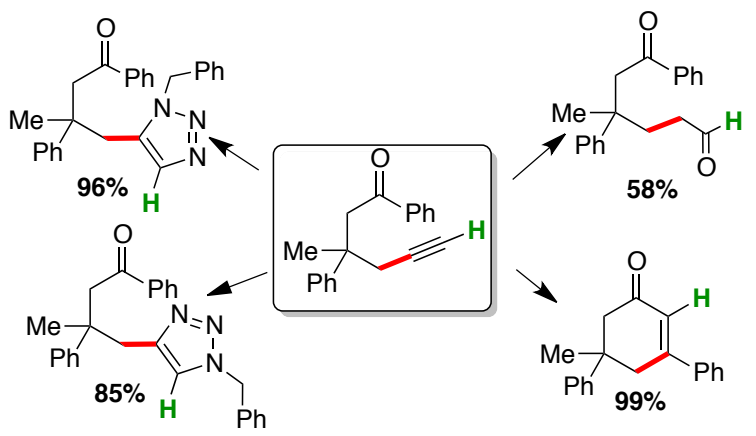

\section{CONCLUSION}

In conclusion, we developed the first route to $\gamma$ alkynylated ketones via Pd-catalyzed C-C bond cleavage. ${ }^{3}$ The reaction is characterized by its wide scope, thus becoming a new platform for molecular diversity.

\section{ACKNOWLEDGEMENTS}

We thank the ICIQ Foundation, the European Research Council (ERC-277883) and MICINN (CTQ2012-34054) for financial support. R.M and A.C thank MICINN for a RyC and JdC fellowship.

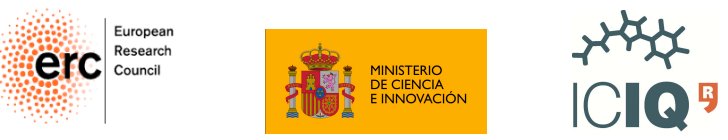

\section{REFERENCES}

${ }^{1}$ For selected reviews, see: (a) Seiser, T.; Saget, T.; Tran, D. N.; Cramer, N. Angew. Chem., Int. Ed. 2011, 7740. (b) Murakami, M.; Makino, M.; Ashida, S.; Matsuda, T. Bull. Chem. Soc. Jpn. 2006, 1315. (c) Aïsa, C. Synthesis 2011, 1315.

${ }^{2}$ Ziadi, A.; Martin, R., Org. Lett. 2012, 1266.

${ }^{3}$ Ziadi, A.; Correa, A.; Martin, R., Chem. Comm. 2013, 4286.

$15^{\text {th }}$ Brazilian Meeting on Organic Synthesis - 15 ${ }^{\text {th }}$ BMOS - November 10-13, 2013 - Campos do Jordão, Brazil 